Pedagogía y Saberes No. 51

Universidad Pedagógica Nacional

Facultad de Educación. 2019. pp. 49-66

\title{
La investigación sobre deserción universitaria en Colombia 2006-2016. Tendencias y resultados
}

\section{Artículo de investigación}

Research about higher education dropout in Colombia 2006-2016. Trends and results A pesquisa sobre deserçao universitária na Colômbia 2006-2016

Marcela Rodríguez Urrego*

Para citar este artículo:

Rodríguez, M. (2019). La investigación sobre deserción universitaria en Colombia 2006-2016. Tendencias y resultados. Pedagogía y Saberes, 51, 49-66.

Profesora de la Universidad Pedagógica Nacional. Estudiante del doctorado en Educación de la Universidad de la Salle (Costa Rica) e investigadora del Grupo Polifonías de la educación comunitaria.

Correo electrónico: smrodriguezu@pedagogica.edu.co

Código oRCID: orcid.org/0000-0002-4230-2539 


\title{
Resumen
}

Este artículo recoge los resultados más relevantes de una revisión documental de 28 investigaciones realizadas en Colombia entre el 2006 y el 2016, sobre deserción en educación superior. Esta revisión fue el punto de partida de una investigación sobre la deserción en la Licenciatura en Educación Comunitaria de la Universidad Pedagógica Nacional. Los resultados se presentan en seis apartados: 1) caracterización de la muestra en la que se tipifican los estudios revisados, según su metodología y objeto de investigación; 2) consideraciones generales sobre la deserción, donde se evidencia la concepción de deserción que sirve de punto de partida de los estudios; 3 ) conceptualización de la deserción, en donde se explicitan los conceptos centrales de los estudios revisados y los problemas asociados a una deficiente conceptualización; 4) factores asociados, en el que se reportan los modelos de deserción utilizados en los análisis estadísticos; 5) presenta de manera sucinta los resultados de las investigaciones empíricas con relación a la deserción en las unidades académicas o conjuntos de ellas estudiados, y 6) un aparte final con resultados y reflexiones referidos a la retención, permanencia y prevención de la deserción.

\section{Palabras clave}

deserción; retención; educación superior; Colombia

\begin{abstract}
This article collects the most relevant results of a documentary review of 28 researches carried out in Colombia between 2006 and 2016, on desertion in higher education. This revision was the starting point of an investigation on the desertion in the Degree in Community Education of the Universidad Pedagógica Nacional. The results presented in six sections: 1) characterization of the sample in which the reviewed studies are classified according to their methodology and research object; 2) general considerations on the desertion where the conception of desertion is evidenced as the starting point of the studies; 3 ) conceptualization of the desertion, where the central concepts of the reviewed studies are explicit and the problems associated with a poor conceptualization; 4) associated factors, in which the models of desertion used in the statistical analyzes are reported; 5) it presents in a succinct way the results of the empirical investigations in relation to the desertion in the academic units or sets of them studied; and 6) a final aside with results and reflections referred to the retention, permanence and prevention of the desertion.
\end{abstract}

\section{Keywords}

desertion; retention; higher education; Colombia

\section{Resumo}

Este artigo reúne os resultados mais relevantes de uma revisão documental de 28 pesquisas realizadas na Colômbia entre 2006 e 2016, sobre a deserção no ensino superior. Esta revisão foi o ponto de partida de uma pesquisa sobre a deserção no programa de graduação de Licenciatura em Educação Comunitária da Universidade Pedagógica Nacional. Os resultados são apresentados em seis seções: 1) caracterização da amostra na qual os estudos revisados são classificados segundo sua metodologia e objeto de pesquisa; 2) considerações gerais sobre a deserção, em que é evidenciada a concepção de deserção que serve de ponto de partida dos estudos; 3) conceituação da deserção, onde os conceitos centrais dos estudos revisados e os problemas associados a uma conceituação pobre são expostos; 4) fatores associados, no qual reportam-se os modelos de deserção utilizados nas análises estatísticas; 5) apresenta rapidamente os resultados das investigações empíricas em relação à deserção nas unidades acadêmicas ou em grupos delas estudados, e 6) uma seção final com resultados e reflexões sobre a retenção, permanência e prevenção da deserção.

\section{Palavras-chave}


Tabla 1

Estudios revisados según método y objeto

\begin{tabular}{|c|c|c|c|c|}
\hline \multicolumn{2}{|c|}{ Estudios revisados } \\
\hline $\begin{array}{c}\text { Cuantitativos } \\
\text { Deserción en }\end{array}$ & 7 & Reflexión teórica & Deserción & Retención \\
\hline Universidades & 4 & Inv. empírica cualitativa & 5 \\
\hline Unidades académicas & 1 & Revisión documental & 2 & 1 \\
\hline Varias universidades & 5 & Sistematización & 2 \\
\hline Orden nacional & 17 & Total & 1 \\
\hline Total & 4 & 11 \\
\hline
\end{tabular}

Fuente: elaboración propia.

Este artículo reporta los resultados más relevantes de la revisión documental realizada para elaborar el marco teórico y operativo del estudio "Comprendiendo la deserción en la Licenciatura en Educación Comunitaria" de la Universidad Pedagógica Nacional. ${ }^{1}$ La selección de la muestra documental se realizó con dos criterios: estudios realizados en Colombia durante la década 2006-2016 y publicados en Internet. Se revisaron 31 documentos de los cuales se seleccionaron 28 como muestra. Para el análisis, cada uno de los documentos fue sintetizado en un RAE y luego disgregado en tres matrices de análisis.

El corpus en cuestión se compuso de cuatro libros, 19 artículos publicados en revistas especializadas, cuatro informes de investigación y una ponencia. Los resultados que se presentan a continuación se agrupan en seis apartados: Caracterización de la muestra según tipo de investigación y objetivos de estudio; Consideraciones generales sobre la deserción, Conceptualización de la deserción, Factores asociados, Resultados reportados y un aparte final sobre retención, permanencia y prevención de la deserción.

\section{Caracterización de la muestra. Tipo de investigación y objeto de estudio}

Las producciones consideradas en esta revisión documental pueden agruparse en dos grandes conjuntos: estudios estadísticos y no estadísticos. Como se observa en la tabla 1, los estudios estadísticos

1 Estudio realizado durante el año 2017 y el primer semestre del 2018 por las profesoras Gloria Chávez Valbuena y Marcela Rodríguez Urrego. constituyen el $61 \%$ de los documentos. Se trata de investigaciones cuantitativas de carácter nacional (cinco estudios ${ }^{2}$ realizados a partir de los datos arrojados por el Sistema de Prevención y Análisis de la Deserción en las Instituciones de Educación Superior - Spadies); institucional (siete estudios toman como unidad de análisis sendas universidades estudiadas en su conjunto, ${ }^{3}$ mientras que tres estudios toman por objeto una o varias unidades académicas dentro de la misma universidad ${ }^{4}$ ) y multi-institucional (un estudio revisa la deserción en varias universidades a partir de una muestra no representativa ${ }^{5}$ ).

Se observa que, de los estudios estadísticos que toman como objeto universidades o unidades académicas, dos son de carácter descriptivo (Malagón, Soto y Eslava, 2007; Rojas y González, 2008); seis, además de describir, indagan por factores de riesgo (Castaño, Gallón, Gómez y Vásquez, 2006; Durán, Pérez-Almonacid, Rodríguez, Reverón y Pinto, 2007; Londoño, 2013; Lopera, 2008; Montes, Almonacid,

2 Dos publicaciones del Ministerio de Educación $(2008,2009)$ y tres del Centro de Estudios Económicos - CEDE- de la Universidad de los Andes (2007; 2014; Sánchez y Márquez, 2012).

3 Universidad de Antioquia (Rodríguez et al., 2013); Universidad Nacional de Colombia (Durán et al., 2007); Universidad Corporación Lasallista (Londoño, 2013); Universidad de los Llanos (Malagón et al., 2007); Universidad de Ibagué (Rojas y González, 2008); Universidad EAFIT (Montes et al., 2010); Universidad Javeriana de Cali (Osorio et al., 2012).

411 programas de la Universidad de Antioquia (Castaño et al., 2006); la facultad de Economía de la Universidad el Rosario (Lopera, 2008); los programas de Psicología de la Universidad San Buenaventura (Mateus et al., 2011) y de Los Libertadores (Ariza y Marín, 2009).

5 Un profesor de la universidad Konrad Lorenz (Medellín, 2010) hace un estudio motivacional con una muestra no representativa de estudiantes de varias universidades. 
Gómez, Zuluaga y Tamayo, 2010; Rodríguez, 2011), y uno establece probabilidades de permanencia o abandono por medio de modelos de supervivencia (Osorio, Bolancé y Castillo-Caicedo, 2012). Los tres estudios realizados desde facultades de psicología incorporan variables psicológicas que dan cuenta de la motivación individual como determinante en la continuidad de los estudios (Ariza y Marín, 2009; Mateus, Herrera, Perilla, Parra y Andersen, 2011; Medellín, 2010). Solo uno de estos estudios clasificados como cuantitativos (Montes et al., 2010) incorpora la dimensión cualitativa.

Los estudios no cuantitativos (11) son de cuatro tipos: reflexión teórica, revisión documental, investigación cualitativa y sistematización. Cuatro toman como objeto la retención o prevención de la deserción (Cardoso-Ortiz, 2011; Pineda-Báez, Pedraza-Ortiz y Moreno, 2011; Suárez-Montes y Díaz-Subieta, 2015; Torres, 2012) y los siete restantes, la deserción.

Los artículos de reflexión teórica giran en torno a: 1) aspectos específicos de la deserción: tensión entre masificación y calidad en la educación superior (Bravo y Mejía, 2010); alfabetización académica y deserción (Olave-Arias, Rojas-García, CisnerosEstupiñán, 2013) o, 2) poblaciones específicas: jóvenes (Barragán y Patiño, 2013; Rojas, 2009); indígenas y afro (Caicedo y Castillo, 2008).

Las revisiones documentales consisten en: una aproximación previa al tema para generar el marco teórico de un estudio específico (Rodríguez, 2011); una revisión para establecer causales de la deserción en América latina y Colombia (Patiño y Cardona, 2015); otra dirigida a identificar elementos significativos para tener en cuenta en un modelo de retención (Torres, 2012) y, una última, vincula el concepto de deserción y el de estrés académico (Suárez-Montes y Díaz-Subieta, 2015).

El estudio clasificado como sistematización realiza la revisión crítica de una estrategia de prevención de la deserción por causas académicas que tomó como estrategia la tutoría entre pares (Cardozo-Ortiz, 2011). Por último, Pineda-Báez, Pérez-Ortiz y Moreno (2011) reportan los resultados de una investigación empírica cualitativa sobre el rol docente en programas de retención de 26 instituciones universitarias.

Observadas las investigaciones en la línea de tiempo, se constata, en el primer periodo 2006-2010, la preponderancia de los estudios cuantitativos (79\%) y disminuye a un $43 \%$ entre el 2011 y el 2016. En este último periodo se evidencia la emergencia de la preocupación por estrategias de retención, ausente en el primer periodo, y una mayor preocupación por dar cuenta de fenómenos asociados al éxito académico.
Estas observaciones son consonantes con la afirmación del Ministerio de Educación (MEN, 2008) sobre la escasez de estudios de deserción en el país antes del 2003 y la preponderante preocupación en un inicio por caracterizar la población desertora, construir índices de deserción y describir de forma estadística el problema en las instituciones.

\section{Consideraciones generales}

Antes de presentar cómo los diferentes estudios conceptualizan y abordan la deserción, debe resaltarse que la división entre estudios cuantitativos y no cuantitativos implica métodos y objetos distintos de investigación y diferencias en la comprensión del fenómeno.

Aunque los estudios estadísticos parten o pueden partir de conceptos precisos sobre lo que sea el fenómeno de la deserción, no abordan con amplitud el contexto estructural o tienen dificultades para operativizar tales variables (elementos fundamentales del contexto como políticas estatales, modelos económicos, dinámicas macro de transformación de la educación y la misma estructura social), de manera tal que, en los resultados terminan obviándose o siendo naturalizadas. Por esta razón Barragán y Patiño (2013) claman por:

[0]bservar la deserción como un problema concreto a solucionar, no como una característica del sistema educativo, donde la baja calidad en los niveles básicos, en países como Colombia, influyen en desventajas académicas de grandes sectores de la población. (p. 57).

En el marco de esta misma preocupación, Rojas (2009) cuestiona la capacidad del abordaje cuantitativo para dar respuestas reales y contundentes al fenómeno, pues "el excesivo consenso cuantitativo de representación de la deserción (...) no es una herramienta institucional eficaz para disminuir el alto impacto social del fenómeno" (p. 85).

En contraste, parte importante de los estudios no cuantitativos abordados plantean como tesis central el carácter estructural de la deserción en la educación colombiana - asentado en factores económicos, culturales y sociales (Barragán y Patiño, 2013; Bravo y Mejía, 2010; Caicedo y Castillo, 2008; Londoño, 2013; Rojas, 2009) - que se refleja en que, por ejemplo, para el 2010, solo el 19.5\% de los jóvenes en edad de estudiar entraron a la universidad y un poco más de la mitad abandonaron sus estudios, lo cual llevó a una oscilación de la deserción entre el 50 y 57\% en la Colombia del siglo XXI (MEN, 2010). Para Barragán y Patiño (2013), se trata de una característica estructural del sistema universitario colombiano, pues 
desde la década de 1990, "la deserción ha mostrado un aumento gradual y constante, sin diferencias significativas entre universidades públicas y privadas o entre personas con pocos o muchos ingresos" (p. 56). Lo que explica que, si bien hay un incremento en la demanda educativa, este no se refleja en la proporción de estudiantes graduados (Lopera, 2008).

La perspectiva económica sobre éxito académico constata un fuerte vínculo entre deserción y capital económico a disposición. El estudio de la Universidad Nacional — UN - reporta, con datos del Departamento Nacional de Planeación (DNP, 2006), cómo, para el 2003 , la cobertura bruta en educación superior alcanzaba, en el quintil más rico de la población, el 73\%, mientras que, en el quintil más pobre, solo llegaba al $7 \%$. Tal inequidad se refuerza por la segmentación jerárquica del campo de la educación superior, que, por ejemplo, favorece el ingreso de la población más pobre a carreras técnicas o tecnológicas, mientras que limita su acceso a la universidad. Dado que las carreras técnicas cuentan con menor reconocimiento social y simbólico, esta segmentación se traduce en discriminación en el futuro campo ocupacional (Durán et al., 2007).

Castro y Rivas (2006, citadas en Londoño, 2013) utilizan el enfoque de la injusticia social para mostrar que las condiciones iniciales de ingreso a la educación superior están fuertemente vinculadas al nivel de renta económica de sus familias. La vulnerabilidad de quienes cuentan con bajos recursos es mayor por dos factores: las limitaciones en el acceso a fuentes de información y la necesidad de trabajar para asistir a clases. ${ }^{6}$

No se trata solo de que las condiciones económicas predigan el logro académico. Sánchez y Otero (2012) argumentan que, además, "la brecha en los puntajes por estrato socioeconómico se ha ido ampliando en el tiempo" (p. 1). Por ejemplo, en el periodo 20082011, "el rendimiento académico de los estudiantes de estratos altos ha mejorado mucho más que el de los estudiantes de estratos bajos". Muestran, además, cómo, las diferencias en el rendimiento académico por cuenta de características socioeconómicas se mantienen en el ámbito universitario, lo que mostraría que la educación universitaria, lejos de disminuir las desigualdades académicas observadas en secundaria y media, las reproduce.

El concepto de capital cultural de Bourdieu (2000) es clave para comprender esta tesis. Si bien desde la década de los setenta, en gran parte de América

6 Afirmación corroborada por diferentes estudios estadísticos revisados (CEDE, 2014; Durán et al., 2007; MEN, 2008; Montes et al., 2010, entre otros).
Latina, se inicia un proceso de masificación de la educación superior, este no se traduce en una participación democrática real de los sectores populares en el sistema educativo, por cuanto estos no cuentan con las condiciones para un buen desempeño (Bravo y Mejía, 2010). Se trata no solo de condiciones económicas sino de otras formas de capital (cultural, social y simbólico), dentro de las cuales el cultural juega un papel determinante para la apropiación del discurso educativo. Este, lejos de ser neutral, es el discurso de la cultura dominante, por lo que refuerza las diferencias académicas (bajos resultados en el Icfes, vacíos formativos en disciplinas específicas, disponibilidad para la formación por necesidad de trabajar, etc.) convirtiéndolas en desventajas en instituciones que hablan en el lenguaje de las élites (Barragán y Patiño, 2013).

Bravo y Mejía (2010) argumentan que detrás de la idea de democratización de la educación manejada por estas reformas poco exitosas (Sánchez y Márquez, 2012) está la idea de aptitud o don personal, que ignora el vínculo entre aptitudes demandadas, valores dominantes y capacidades asociadas al capital económico (formas de estructurar el pensamiento, tipos de conocimiento, entrenamiento en formas determinadas de evaluación, etc.), que los miembros de las clases altas han tenido mayores posibilidades de desarrollar.

Más grave aún, Barragán y Patiño (2013) muestran cómo tal masificación educativa al no estar respaldada económicamente desde el Estado, revirtió en una reglamentación que reduce sus funciones a la vigilancia y el control, promoviendo la creación de un mercado paralelo sostenido por ofertas de bajo nivel que suplen la incapacidad del Estado de financiar la educación superior, con una combinación de baja calidad y crédito y con un impacto mínimo en la calidad de la vida laboral a la que puede acceder el nuevo profesional. Se mantiene así la segmentación del mercado laboral mientras que las ofertas de educación superior de baja calidad sostienen la permanencia de la cuasi-estamental sociedad colombiana.

Caicedo y Castillo (2008), en su reflexión sobre las dificultades que enfrentan indígenas y afrodescendientes en su ingreso a la educación terciaria, enfatizan el carácter eurocéntrico de la Universidad, uno de cuyos objetivos es la naturalización de los ideales liberales y de los valores modernos e ilustrados que dificultan hacer realidad las aspiraciones interculturales y las demandas de pluralismo epistémico de los grupos étnicos referidos. En tanto se trata de un proyecto epistemológico que busca absolutizar la idea de conocimiento como ciencia, la Universidad impide el reconocimiento de las epistemologías propias de estas poblaciones, con lo cual subvalora sus 
formas de vida y sus saberes ancestrales en contra del artículo 68 de la Constitución Política. ${ }^{7}$ Las cifras son escandalosas: el promedio nacional de ingreso a la educación superior es del 37\%, para las poblaciones afrocolombianas es del 33\% y para las indígenas el $20 \%$. Pero mientras que un $50 \%$ de los estudiantes deserta de la educación superior, el promedio indígena es del 80\% (Caicedo y Castillo, 2008).

La baja flexibilidad de la universidad frente a la diversidad cultural y el limitado acceso y permanencia de poblaciones vulnerables se ven reforzados por las políticas neoliberales en curso, desde la década de los 80. Aunque la Constitución de 1991 reconoce la autonomía universitaria, en virtud de las transformaciones en el financiamiento de las universidades públicas, la garantía estatal del derecho a la educación se ha visto restringida y lleva a perversas modificaciones en el lenguaje que rebautizan la equidad en términos de mercado. Así argumenta la Asociación Nacional de Universidades - ASCUN (2007, p. 18): “La igualdad (empieza a ser) entendida como factor de la oferta de bienes y servicios, y la equidad, como factor de la demanda" (citado en Caicedo y Castillo, 2008, p. 67), por lo que la universidad queda confinada al marco del mercado educativo y se ocupa en competir y ser competitiva.

Rojas (2009) argumenta también desde la óptica de las dinámicas culturales poco dialógicas cómo, a los factores macroeconómicos, se suma la incapacidad de las instituciones de educación superior de profundizar en el conocimiento sobre el estudiante de secundaria y de poner en diálogo estas informaciones con sus perfiles de egreso. Para este autor, el mayor problema de la crisis universitaria es la baja calidad de la educación, que se refleja en "modelos pedagógicos y de evaluación inadecuados, planes de estudio que no cumplen las expectativas del educando y excesiva rigidez en la manera de conducir la academia, estilos docentes y administrativos poco amistosos desde la perspectiva del estudiante" (p. 82). Barragán y Patiño (2013) suscriben esta interpretación afirmando que "la educación superior no parece constituir un sistema de referencia social para los jóvenes" (p. 61) más aún cuando sus promesas de ser camino expedito para consecución de empleo, mejora de la calidad de vida, y beneficio comunitario parecen incumplirse sistemáticamente.

7 Este reza: "Los integrantes de los grupos étnicos tendrán derecho a una formación que respete y desarrolle su identidad cultural." (Constitución Política de Colombia, 1991)

\section{Conceptualización de la deserción}

El estudio de la deserción estudiantil en educación superior presenta dificultades, no siempre explicitadas con respecto a la definición del fenómeno. Un aspecto señalado por muchas de las investigaciones que se abocan a una mirada cuantitativa tiene que ver con su carácter "elusivo" y complejo, razón por la cual algunas se abstienen de una aproximación conceptual, y maniobran de forma directa con una operativa que establece características como fenómeno susceptible de ser medido (Durán et al., 2007).

La insuficiente definición conceptual lleva, según Tinto (1975, citado en Montes et al., 2010), a que bajo el nombre de deserción se agrupen diversas formas de retiro estudiantil, por ejemplo, deserción por causas académicas y aquella de carácter voluntario; o, retiro temporal y retiro permanente. La insuficiente definición lleva a la inconmensurabilidad de los resultados del análisis estadístico de diversos estudios pues las mediciones no responden a los mismos fenómenos. Para este autor, el problema de la definición responde a una elección que debe estar motivada por la identificación, entre las numerosas formas de abandono, (de aquellas) que merecen una intervención institucional, pues no toda deserción lo merece (1989, en Olave-Arias et al., 2013).

Si bien algunos estudios revisados no explicitan su concepto de deserción, todos pueden considerarse expresados en las reflexiones de Castaño y sus colegas (2006), quienes proponen: "La deserción es un evento individual de interrupción o desvinculación de la trayectoria académica institucional resultado de uno o varios procesos en el plano personal, institucional o social." (p. 48).

El peso dado a factores externos o internos constituye una de las diferencias entre los estudios. A los factores externos se los agrupa como "modelo de desgaste" (Bean, 1980, citado en Osorio et al., 2012, p. 33) mientras que los que dan preponderancia a los factores internos se los conoce como modelo de integración (Tinto, 1975 y Spady, 1970, citados en Osorio et al., 2012).

Un ejemplo de la comprensión de la deserción desde el modelo de desgaste se encuentra en la definición propuesta por Tinto (1982) y Giovagnoli (2002) (citados en MEN 2008, p. 18 y 2009, p. 22) quienes conciben la deserción como la "situación a la que se enfrenta un estudiante cuando interrumpe y no logra concluir su proyecto educativo". El modelo de integración se ejemplifica en la definición de Fishbein y Ajzen (1975, en CEDE, 2014, p, 10) quienes plantean 
la deserción como resultado del debilitamiento de las intenciones iniciales del estudiante, influido por su auto-concepto, lo que supone que cuanto mayor sea la integración y adaptación del estudiante a la vida universitaria, mayor será su compromiso y menor la probabilidad de desertar.

El estudio de la Universidad Nacional propone una concepción de deserción que evidencia su multicausalidad y la necesidad de integrar "la trayectoria académico institucional" del estudiante y "las causas y consecuencias propias del plano institucional y social" (Durán et al., 2007, p. 48). En este sentido, Tinto (1982, citado en Durán et al., 2007) plantea tres niveles desde los cuales puede observarse la deserción: el nacional, el institucional y el individual, cada uno con una definición y forma de abordaje específicas, limitándose así las posibilidades de una definición universal.

El fenómeno de la deserción es un fenómeno situado en el que se entrecruzan factores macroeconómicos y de política educativa estatal con factores institucionales (que incluyen consideraciones sobre excelencia académica; educación como servicio o derecho; objetivos mismos de la formación académica; patrones de relacionamiento entre estudiantes, profesores y administrativos, entre otros), factores sociales (capital cultural, social y económico al que tienen acceso los estudiantes) y factores de orden individual (motivación, resistencia a la frustración, historia personal y familiar, proyecto de vida, etc.). Así, la deserción dista de ser un fenómeno homogéneo por lo que sus estudiosos establecen precisiones conceptuales para profundizar su comprensión.

La primera precisión conceptual necesaria es establecer la diferencia entre deserción o desvinculación voluntaria (cuando el estudiante decide abandonar sus estudios) y desvinculación forzada (cuando el estudiante suspende sus estudios por razones estipuladas en la institución, como requerimientos académicos mínimos no alcanzados por el estudiante) (Castaño et al., 2006).

Considerada desde el punto de vista de su temporalidad, la deserción puede ser de tres tipos: precoz (situación en la cual el estudiante, a pesar de haber obtenido la admisión en la universidad, no se matricula), temprana (abandono de los estudios en los primeros semestres del programa) y tardía (abandono de los estudios en los últimos semestres) (Durán et al., 2007; Lopera, 2008; Montes et al., 2010).

Con respecto al espacio, se consideran la deserción institucional: cuando el estudiante abandona la institución; y, la deserción interna o del programa académico: cuando el estudiante realiza una transferencia a otro programa de la misma institución. Algunos estudiosos plantean que, en sentido estricto, la interrupción de un programa curricular por traslado a otro de la misma institución no es deserción sino traslado interno. Se hablaría entonces de traslado externo si el estudiante se retira, pero se matricula en una institución diferente. Desde el punto de vista nacional este fenómeno no clasificaría como deserción pues los estudiantes continúan dentro de la educación superior. La dificultad para la medición precisa de estos fenómenos radica en el seguimiento insuficiente de los traslados estudiantiles institucionales (Durán et al., 2007; Lopera, 2008; MEN, 2008; Montes et al., 2010; Vásquez, Castaño, Gallón y Gómez, 2003).

Dos definiciones también importantes son los conceptos de cobertura real: proporción de estudiantes que logran graduarse en el tiempo previsto institucionalmente y rezago: que caracteriza una trayectoria académica cuya duración es mayor a la establecida por la institución para la obtención del grado (Durán et al., 2007). Estos conceptos evidencian la diferencia entre el retiro permanente, que corresponde a la deserción o abandono y la desvinculación temporal, que implica retorno (Durán, et al., 2007), retiro parcial (Rojas, 2009) o deserción de corto plazo (Osorio et al., 2012).

Las aproximaciones a la desvinculación temporal no son consistentes entre sí, para autores como Rojas (2009) se trata de una forma potencial de deserción pues prolonga la consecución del grado y aumenta la probabilidad de abandono. Por el contrario, el estudio de la Universidad Nacional advierte que el fenómeno varía según su contexto. Por ejemplo, en virtud de la subsidiaridad de su matrícula, se encuentra más extendido en las universidades públicas. En el contexto de la Universidad Nacional de Colombia se presenta, en la mayoría de los casos, como factor protector de la deserción y está asociado a una doble vulnerabilidad (económica y académica) y/o a la existencia de cambios vitales (matrimonio o paternidad, entre otros). No obstante, a mayor duración de la desvinculación, mayores probabilidades de deserción. Por su parte, Osorio y sus colegas (2012) basándose en un estudio de logit multinomial (Stratton, 0'Toole y Wetzel, 2008, en Osorio et al.), afirman que los factores que llevan a la deserción temporal no coinciden con aquellos que llevan a la deserción definitiva.

Otra diferencia conceptual importante es introducida por el estudio de la Universidad Javeriana sobre retención estudiantil (Torres, 2012). Según la autora, estudios previos utilizan indistintamente los términos retención y persistencia estudiantil. Sin embargo, se trata de dos enfoques diferentes: la persistencia está relacionada con la habilidad del estudiante o la 
motivación para continuar y terminar sus estudios; la retención es una condición institucional asociada a la búsqueda de garantizar la permanencia de los estudiantes matriculados. Se marca así la diferencia entre la perspectiva del estudiante y la institucional frente al fenómeno de la permanencia. Ahora bien, ya que la persistencia estudiantil tiene que ver con "el deseo y la acción" de permanecer, pareciera una condición solo individual, pero un análisis de mayor profundidad evidencia que se trata de condiciones contextuales: "la persistencia es la capacidad académica, las características sociales y la motivación de un estudiante para alcanzar sus metas educativas." (Torres, 2012, p. 29).

Se puede conceptualizar la retención institucional como "la proporción de estudiantes que siguen estando matriculados en la misma institución de año a año"; la retención del sistema como la medida que "considera a los estudiantes que salen de una institución y se matriculan en otra; es decir, tiene en cuenta las transferencias del estudiante hasta que obtiene su título" (p. 26) y, la retención dentro de una especialidad o disciplina que refiere a la continuidad dentro de un área de estudio.

Por su parte, las reflexiones e investigaciones sobre retención echan mano de conceptos o perspectivas específicas para articular sus propuestas. Por ejemplo, Olave-Arias y sus colegas (2013) centran su interés en las habilidades lecto-escriturales específicas del nivel universitario comparándolas con aquellas demandadas en la educación secundaria. Por su parte, CardozoOrtiz (2011) se centra en la estrategia de tutoría entre pares como factor protector de la deserción por causas académicas. Pineda-Báez y colegas (2011) centran su estudio en la comprensión del rol docente en las dinámicas de retención y deserción. Factor destacado por Londoño (2013), quien establece responsabilidades institucionales en la deserción fruto de las limitaciones en la calidad de la educación que ofertan y del ausentismo profesoral provocado por modalidades de contratación que limitan los aportes docentes a la conformación de comunidades académicas activas. Este mismo autor plantea como otro factor relevante la escasa orientación vocacional de los estudiantes y la omisión, en ella, de las habilidades relevantes para el campo de saber.

\section{Factores asociados}

Dentro de los estudios estadísticos, algunos establecen cadenas de causalidad que dan cuenta de "factores asociados", "de riesgo" o "determinantes" de la deserción y modelan sus dinámicas en un contexto específico. Donoso y Schiefelbein (2007, citados en Montes et al., 2010) clasifican los abordajes de la deserción en cinco, a partir de las disciplinas en las que se desarrollan: psicológico, sociológico, económico, organizacional e interaccionista. La tabla 2 presenta un resumen de los énfasis particulares de cada enfoque.

Tabla 2

Enfoques en el estudio de la deserción

\begin{tabular}{c|l} 
Nombre & \multicolumn{1}{c}{ Enfoque } \\
\hline Psicológico & $\begin{array}{l}\text { A partir de variables individuales como creencias y actitudes sobre terminar un programa académico y } \\
\text { las consecuencias de no hacerlo establece diferencias de perfil entre quienes abandonan y quienes cul- } \\
\text { minan. Los modelos explican sobre esta base si un estudiante desertará o no. (Ethington, 1990) }\end{array}$ \\
\hline Sociológico & $\begin{array}{l}\text { Este enfoque (Spady, 1970) incluye las variables psicológicas en el marco de la teoría del suicidio de } \\
\text { Durkheim. El programa o universidad se entiende como un sistema con valores y estructura social pro- } \\
\text { pios, que espera del estudiante su acoplamiento. La integración depende de la aceptación de las nor- } \\
\text { mas y valores de la comunidad. El "suicidio" se da cuando entorno y situación se vuelven tan incómodos } \\
\text { que el estudiante decide abandonarlos. En este enfoque son claves la integración con compañeros y } \\
\text { profesores, el aprecio por los valores institucionales y la satisfacción del estudiante con la experiencia } \\
\text { universitaria. También se incluyen variables socioeconómicas, del ambiente institucional y del pasado } \\
\text { familiar y cultural del desertor. }\end{array}$ \\
\hline Económico & $\begin{array}{l}\text { La deserción es fruto de un análisis costo-beneficio realizado por el individuo en el que se incluyen } \\
\text { variables financieras. Aunque en la misma lógica podrían considerarse otros aspectos (satisfacción con } \\
\text { el programa o valoración que tenga el individuo del conocimiento y del uso del tiempo) el modelo no } \\
\text { los contempla. Este enfoque logra predecir con acierto la deserción de estudiantes de escasos recursos, } \\
\text { pero no explica la deserción en otros casos. Es desarrollado por Donoso y Schiefelbein (2007). }\end{array}$
\end{tabular}




\begin{tabular}{|c|l}
\hline Nombre & \multicolumn{1}{c}{ Enfoque } \\
\hline $\begin{array}{c}\text { Organiza- } \\
\text { cional }\end{array}$ & $\begin{array}{l}\text { Este enfoque toma como eje central la institución académica al considerarla como el factor más influ- } \\
\text { yente en la decisión del estudiante sobre la deserción, la cual se lee a partir de las características de la } \\
\text { institución: los servicios que presta a los estudiantes. Además de tomar en consideración la historia } \\
\text { del individuo y su capacidad de "sobrevivir", incluye características como calidad de la docencia y ser- } \\
\text { vicios extracurriculares que presta la institución. La ventaja de este análisis es que sus resultados pue- } \\
\text { den apoyar la toma de decisiones para ajustar las políticas institucionales, atacando el problema de la } \\
\text { deserción desde su raíz. Este modelo es desarrollado por Bean (1980), Vásquez, Gallón y Gómez (2003) } \\
\text { y ceDE (2007). }\end{array}$ \\
\hline Interac- & $\begin{array}{l}\text { Reconoce la interacción entre las variables explicativas del modelo sin dar preponderancia a ninguna } \\
\text { de ellas. Parte de evidenciar los problemas que genera una escasa definición del concepto de deserción } \\
\text { y la interpretación limitada que emerge si no se tienen en cuenta las variables correctas. Considera } \\
\text { variables institucionales y sociales y asume que los estudiantes realizan de manera permanente análisis } \\
\text { costo-beneficio sobre las implicaciones de ser estudiante y, en ocasiones, toman la decisión de desertar } \\
\text { cuando los costos se evidencian mayores que los beneficios. Factores institucionales negativos y facto- } \\
\text { res personales que limiten la adaptación al ambiente del programa universitario llevan a un aumento } \\
\text { de los costos del estudiante. Por otro lado, factores institucionales valorados como positivos junto con } \\
\text { la remuneración futura proveniente de su educación serán entendidos como beneficios. El modelo es } \\
\text { longitudinal por cuanto sigue al estudiante desde su ingreso hasta su salida, sea por graduación o deser- } \\
\text { ción. Según su autor (Tinto,1987), si bien permite explicar la deserción de una institución universitaria, } \\
\text { el modelo no da cuenta de la salida total del sistema de educación superior. }\end{array}$ \\
\hline
\end{tabular}

Fuente: elaboración propia a partir de Montes et al. (2010).

Los estudios explicativos toman en consideración cuatro aspectos como determinantes de la deserción: individuales, académicos, socioeconómicos e institucionales. La tabla 3 presenta un resumen de las variables encontradas en cada uno de ellos.

Cada uno de los modelos presentados incorpora solo algunas variables y deja de lado otras. El modelo más completo y referenciado en la literatura consultada es el interaccionista de Tinto (1987). Vincula variables propias de la institución y condiciones particulares del estudiante integrando diversos aspectos relevantes desarrollados en el campo de investigación.

En los diferentes estudios revisados se observó que la aplicación de estos modelos en el contexto nacional no depende solo de reflexiones teóricas, sino, de la disponibilidad de información con respecto a los

Tabla 3

Aspectos considerados en los estudios de deserción y algunas variables asociadas

\begin{tabular}{|c|c|c|c|}
\hline Individuales & Socioeconómicos & Académicos & Institucionales \\
\hline $\begin{array}{c}\text { Edad } \\
\text { Género } \\
\text { Estado civil } \\
\text { Vivienda (propie- } \\
\text { dad y educación) } \\
\text { Calamidades } \\
\text { Expectativas sobre la Uni- } \\
\text { versidad y la carrera } \\
\text { Embarazos } \\
\text { Número de hermanos } \\
\text { Posición dentro de } \\
\text { los hermanos } \\
\text { Incompatibilidad horario }\end{array}$ & $\begin{array}{c}\text { Estrato } \\
\text { Dependencia } \\
\text { Personas a cargo } \\
\text { Educación y ofi- } \\
\text { cio de los padres } \\
\text { Crecimiento PIB } \\
\text { Vinculación laboral } \\
\text { Situación laboral de } \\
\text { estudiante y padres } \\
\text { Conectividad } \\
\text { Acceso a prés- } \\
\text { tamo estudiantil } \\
\text { Uso de servicios de bien- } \\
\text { estar universitario } \\
\text { Tamaño grupo familiar } \\
\text { Número de apor- } \\
\text { tantes al hogar } \\
\text { Valor pensión }\end{array}$ & $\begin{array}{c}\text { Tipo de colegio } \\
\text { Inicio inmediato o mediato } \\
\text { Puntaje Icfes } \\
\text { Promedio } \\
\text { Experiencia acadé- } \\
\text { mica anterior } \\
\text { Puntaje en examen de } \\
\text { estado por áreas } \\
\text { Desempeño acadé- } \\
\text { mico por áreas } \\
\text { Número de herma- } \\
\text { nos universitarios } \\
\text { Sede y carrera a las que } \\
\text { se está vinculado } \\
\text { Deserciones previas } \\
\text { Orientación profesional } \\
\text { Métodos de estudio }\end{array}$ & $\begin{array}{c}\text { Servicios de bienestar } \\
\text { Relación con profesores } \\
\text { Relación con compañeros } \\
\text { Instalaciones } \\
\text { Solvencia pedagó- } \\
\text { gica de los docentes } \\
\text { Actividades } \\
\text { extracurriculares } \\
\text { Programas de apoyo } \\
\text { académico } \\
\text { Programas de apoyo } \\
\text { financiero } \\
\text { Compromiso con los valo- } \\
\text { res institucionales }\end{array}$ \\
\hline
\end{tabular}

Fuente: elaboración propia. 
estudiantes inscritos. Esto se hace aún más relevante si se considera que la confiabilidad de estos estudios depende de su realización tras el hecho cumplido, bien de la deserción, bien de la graduación. Al respecto, Durán y sus colegas (2007) afirman que la "deserción corresponde a la desvinculación definitiva" y por tanto no puede "conocerse con certeza pues nunca se verificará la trayectoria completa del estudiante: si vuelve en 10 años, si volverá a estudiar en otra universidad, etc.; así, se puede dar cuenta solo de que vuelva o no" en los semestres de estudio que establezca la investigación (p. 50). Para la recolección de información esto implica un proceso que debe haberse efectuado por lo menos siete años antes de la realización del estudio.

Cada indagación cuantitativa sobre la deserción hace uso de un modelo estadístico específico para cuantificarla. El análisis estadístico se diferencia entre estudios de marco estático y estudios de supervivencia, longitudinales o de duración. Según la revisión documental realizada por los estudios del MEN (2008), en Colombia, en general, se ha asumido un "marco estático", que prioriza la explicación de la razón del abandono y omite el proceso mismo: su evolución longitudinal y la interacción entre las diferentes categorías.

Los estudios de supervivencia, duración o longitudinales, que se conocen también como modelación del riesgo, describen y explican longitudinalmente la deserción, en la búsqueda de identificar las probabilidades de que un alumno abandone o se gradúe en la universidad. Esta consideración de la dinámica del proceso de deserción compara la probabilidad de abandono de los estudios en distintos momentos de la vida académica, y muestra que existen periodos críticos en la trayectoria de un estudiante, en los que las interacciones entre condiciones institucionales e individuales influyen de forma directa en la deserción (MEN, 2008).

\section{Resultados reportados}

\section{Aspectos individuales}

Tomando como referencia los aspectos enunciados arriba, los estudios revisados establecen características asociadas a, o determinantes de la deserción. Con relación a los factores individuales, el género es uno de los más significativos. La mayor parte de los estudios considerados reportan mayor riesgo de deserción en hombres que en mujeres. Los estudios del MEN precisan la probabilidad de supervivencia de las mujeres en $73,5 \%$ frente a $68,6 \%$ de los hombres. Por su parte, el estudio de la Universidad de los Llanos (Malagón et al., 2007) constata que mientras los factores académicos son la principal causa de deserción en los hombres (32,4\% frente a un 15,4\% de las mujeres), los factores individuales (embarazos y problemas familiares) tienen mayor incidencia en las mujeres (11,8\% frente a un $9.8 \%$ de los hombres).

En relación con la edad como predictor de la deserción, los resultados son menos uniformes. Para el nivel nacional, el estudio del MEN (2009) afirma: "los estudiantes que ingresan al sistema a una mayor edad acumulan tasas de deserción por cohorte $17 \%$ más altas de aquellas que presentan los estudiantes más jóvenes" (p. 92), y atribuye tal diferencia a compromisos laborales, familiares y económicos que se incrementan con la edad. Además, la probabilidad de deserción tardía es mayor para un estudiante maduro que para uno joven. ${ }^{8}$ Algunos estudios en universidades y programas específicos no llegan a los mismos resultados: la Universidad EAFIT (Montes et al., 2010); la Universidad de Ibagué (Rojas y González, 2008); Los Libertadores (Ariza y Marín, 2009) y la Javeriana de Cali (Osorio et al., 2012) no encuentran diferencias significativas en la deserción por rango de edad. El estudio de la Universidad de Antioquia encuentra que, si bien, la edad de inicio incide en el riesgo de desertar "este decrece marginalmente con el aumento de la misma" (p. 20). ${ }^{9}$ En el caso de la Universidad de los Llanos (Malagón et al., 2007) se reporta mayor incidencia de la deserción entre los 22 y 24 años (44\%), seguido de un 25\% entre los 19 y 21 años. La menor incidencia está entre los 25 y 30 años. Las diferencias en estos resultados son fruto de variaciones contextuales, lo que confirma el carácter situado del fenómeno de la deserción.

Otros factores de riesgo individuales reportados por los estudios revisados son: vinculación al mercado laboral, estar casado, tener un mayor número de hermanos. Su incidencia es variable en función del contexto. El estudio de la Universidad del Rosario reporta como relevante el lugar de procedencia (Lopera, 2008). Otros estudios reportados por Patiño y Cardona (2012) evidencian, además, la importancia de "la biografía personal y académica (...), el consumo de sustancias psicoactivas (...), las expectativas personales insatisfechas, relaciones intrafamiliares, motivación, problemas de salud, temperamento, apatía, tendencia a la depresión, ausencia de perspectiva de futuro e incompatibilidad con valores institucionales" (p. 12).

8 Afirmaciones consistentes con los análisis del CEDE (2014), la Universidad del Rosario (Lopera, 2008), la Universidad Nacional (Durán et al., 2007) y la Universidad de Antioquia (Rodríguez, Posada, Estrada y Velázquez, 2013).

9 Aseveración similar hace el estudio del CEDE (2007). 
Por otro lado, el estudio desde una perspectiva psicológica de la Universidad de San Buenaventura (Mateus et al., 2011) plantea que:

el $50 \%$ de los participantes se vio enfrentado a problemas de índole personal durante su proceso formativo (...): inseguridad de asumir la carrera, dificultades de salud, falta de motivación e inconformidad con la carrera, incompatibilidad de horarios de estudio y trabajo, muerte de familiares y, en un caso, embarazo (p. 130).

\section{Aspectos socioeconómicos}

Con respecto a las características socioeconómicas, los estudios de carácter nacional (CEDE, 2007, 2014; MEN, 2008, 2009) refieren como factores de riesgo: nivel familiar de ingresos; situación laboral en el momento de presentar el Icfes (quienes trabajaban en ese momento están 10 puntos por encima en el riesgo de desertar que quienes no lo hacían); el nivel educativo de la madre (la diferencia de riesgo entre un estudiante cuya madre tiene como máximo nivel educativo primaria y una con nivel universitario es también de 10 puntos); vivir "en arriendo" (aunque la diferencia de riesgo comparado con quienes son propietarios de la vivienda que habitan es de solo 3 puntos); el número de hermanos (quienes no tienen hermanos presentan una tasa de deserción 10 puntos por debajo de quienes tienen más de cuatro).

De este modo, un estudiante que trabajaba al momento de presentar el Icfes, carece de vivienda propia y tiene madre con bajo nivel educativo, tiene una probabilidad de desertar del $60 \%$, mientras que un estudiante que no trabaja, cuenta con vivienda propia y madre con nivel de formación universitario ve reducida su probabilidad de deserción al $42 \%$. Si bien este casi $20 \%$ de diferencia es significativo y constata las grandes dificultades que enfrentan los jóvenes de estratos socioeconómicos bajos para llevar a cabo sus estudios, en la interpretación del fenómeno de la deserción es importante no perder de vista el altísimo $42 \%$ de personas que tienen condiciones económicas y culturales, pero deciden no continuar sus estudios. Estos datos confirman la tesis de Rojas (2009) y Barragán y Patiño (2013) sobre la pérdida del encanto del estudio para los jóvenes colombianos.

Indicadores macroeconómicos como tasas de desempleo y nivel de ingresos departamentales muestran su influencia negativa en la permanencia de los más pobres. Como afirma el estudio de la Universidad Nacional (Durán et al., 2007): "contar con ciertas condiciones de entrada hace probable una trayectoria académica".
Por otro lado, el citado estudio muestra que el trabajo estudiantil remunerado dentro de la Universidad es un factor de retención. Un estudiante en estas condiciones tiene una probabilidad máxima de desertar del $21,7 \%$, mientras que "un estudiante que no haya trabajado tiene una probabilidad máxima de 75,9\%" (p. 159). Este efecto desaparece cuando el trabajo es fuera de la universidad. El estudio evidencia también que el préstamo estudiantil disminuye la probabilidad de deserción, aunque aumenta las probabilidades de rezago.

Los estudiantes indígenas tienen mayores probabilidades de rezago que el resto de los estudiantes regulares, en función de dinámicas culturales y económicas que requieren el retorno regular, a veces por largos periodos, al lugar de origen (Durán, et al., 2007).

Los resultados del estudio de la Corporación Lasallista (Londoño, 2013) muestran, además, otras expresiones del vínculo entre condiciones socioeconómicas adversas y deserción. Por ejemplo, quienes atraviesan dificultades económicas tienen un 48\% de probabilidades de desertar al igual que quienes vienen de hogares con padres separados. Quienes pertenecen a estratos 2 y 3 ven sus probabilidades de desertar aproximarse a un $71 \%$.

\section{Aspectos académicos}

El documento "Deserción Estudiantil en la Educación Superior Colombiana" (MEN, 2009) afirma que la implementación del Spadies evidenció que "las condiciones académicas de entrada pueden llegar a ser determinantes para pronosticar las probabilidades de deserción de un joven matriculado en un programa de educación superior" (p. 86). Esta afirmación pone de cabeza la mayoritaria percepción previa sobre un mayor impacto de las situaciones financieras y económicas. Si bien las diferencias en el nivel de ingresos familiares explican en parte el riesgo de deserción, la consideración de variables de rendimiento académico, como el puntaje del Icfes, es mucho más potente para explicarla y, por lo tanto, prevenirla. Así, la diferencia en el riesgo de deserción a décimo semestre entre quienes obtuvieron puntaje bajo (57\%) y puntaje alto (35\%) en el Icfes es de 22 puntos (p. 88). Razón por la que, sin desconocer su vínculo con las condiciones socioeconómicas, se afirma la mayor relevancia de los aspectos académicos. De allí la necesidad de fortalecer académicamente a los estudiantes para lograr mayor permanencia estudiantil y mayor equidad para quienes acceden al sistema como primera generación. 
Esta apreciación es consistente con los resultados del estudio de la Universidad EAFIT (Montes et al., 2010) que identifica que las condiciones académicas de entrada son relevantes (estudiantes con bajo puntaje en Icfes, procedentes de colegios con rendimiento académico inferior o muy inferior requieren mayor atención por las debilidades académicas con las que ingresan). En general, quienes desertan tienen promedios más bajos y cancelan más materias. En muchos casos, las ciencias básicas se convierten en filtro académico.

En esta universidad, la deserción temprana está asociada en particular a variables académicas y limitaciones en la elección del programa de estudios. Los factores explicativos de la deserción son, en orden de importancia: el promedio semestral, el contar con una beca (mientras que un $68.5 \%$ de quienes no estuvieron becados desertó, solo un $14.1 \%$ de los, alguna vez, becados lo hizo), el número de créditos o materias perdidas, la edad y el puntaje del Icfes. El estudio desarrolla modelos para la deserción en cuarto y séptimo semestre y encuentra, que, en cuarto, los factores determinantes son: promedio del semestre y número de créditos perdidos (por cada décima que se aumente el promedio, la probabilidad de desertar disminuye en $1,85 \%$, mientras que, si pierden una materia la probabilidad aumenta en $1.2 \%$ ), habiendo desaparecido el efecto de género, colegio y beca. Para séptimo semestre, el único factor predictor encontrado es el promedio académico acumulado.

El estudio de la Universidad Nacional (Durán et al., 2007) establece parte de sus resultados en términos de factores protectores: se trata de "condiciones estructurales, individuales y del proceso universitario" (p.115) que permiten un ajuste entre estudiante y universidad, que redunda en graduación. De las cuatro características principales asociadas al perfil protector, dos tienen que ver con la dimensión académica: ${ }^{10}$ el estudiar carreras con bajas tasas de absorción y el tener una baja vulnerabilidad académica y económica. Quienes caen dentro de este perfil tienen probabilidades de grado por encima del $70 \%$ y la mitad de ellos ve aumentadas sus probabilidades de éxito académico por encima del $80 \%$.

Con relación al primer aspecto, el estudio establece, en consonancia con estudios previos, que existen ciertas facultades con promedios menores de deserción (salud, derecho, artes y ciencias económicas) asociados a: alta flexibilidad en el plan curricular (el estudiante puede ajustar asignaturas en términos

10 Las otras dos son: ser mujer de 18 años y lograr una mayor integración social e institucional. de intereses y disponibilidad de tiempo) y bajas diferencias en la promoción de estudiantes con diferentes grados de vulnerabilidad académica y económica.

El CEDE (2014) plantea, sin profundizar en ello, diferencias a nivel nacional en la deserción según programa. Se dan mayores niveles de deserción en programas pertenecientes a ciencias de la educación, ingenierías y arquitectura y, aún más en las IEs técnicas o tecnológicas. Para instituciones universitarias y universidades el mayor riesgo de deserción se presenta en programas del área de matemáticas y ciencias naturales.

Por su parte, el estudio de la Corporación Universitaria Lasallista (Londoño, 2013) identifica que factores académicos, como el uso esporádico de métodos de estudio y el no buscar orientación psicopedagógica, son factores de riesgo de altísima incidencia (64\% y $87 \%$ respectivamente).

Desde una perspectiva cualitativa, el estudio sobre los efectos de la alfabetización académica en la deserción (Olave-Arias et al., 2013) argumenta de forma convincente sobre las diferencias entre estilos de aprendizaje y argumentación en la educación media y la superior. Como lo muestra la tabla 4, se evidencian contrastes fundamentales entre textos, finalidades, tipos de ejercicio y habilidades requeridas en los procesos lecto-escriturales. Contrastes, para los cuales, en muchos casos, las mismas universidades omiten preparar o lo hacen de manera insuficiente, por lo que estas competencias básicas pueden transformarse en fuente de frustración y posterior deserción.

Barragán y Patiño (2013) en su argumentación sobre el carácter estructural de la deserción en Colombia presentan la vulnerabilidad académica del estudiante universitario como función de la baja calidad de su formación básica. Esto se evidencia en los resultados de la prueba Pisa, según los cuales entre el $40 \%$ y el $60 \%$ de los estudiantes latinoamericanos de secundaria no alcanzan los niveles de rendimiento requeridos para el acceso a la universidad. Señalan, además, con base en diferentes estudios, que las "grandes carencias en competencias comunicativas, analíticas, de síntesis y conceptualización, además de pobre formación en matemáticas, ciencias naturales y sociales y en lenguas extranjeras (...) afectan en mayor medida a estudiantes de menores ingresos y provenientes de municipios pobres" (p. 52).

\section{Aspectos institucionales}

El estudio nacional del CEDE (2014) al revisar aspectos institucionales referidos a la deserción, encuentra diferencias importantes según se trate de instituciones oficiales o privadas: las IEs técnicas oficiales 
Tabla 4

Diferencias implicadas en la lectura y la escritura entre nivel escolar y universitario

\begin{tabular}{|c|c|c|}
\hline & Nivel escolar & Nivel universitario \\
\hline Conocimiento & General & Particular \\
\hline Enunciatario & Estudiante & Especializado \\
\hline Estilo & Cotidiano & Largo \\
\hline Extensión & Corto & Expositiva y argumentativa \\
\hline Secuencia textual & Narrativa (predominante) & Académico-científico \\
\hline Género discursivo & Académico-instruccional & Libros y artículos especializados \\
\hline Fuentes & Libro de texto & Anélisis y relaciones intra \\
\hline Finalidad de lectura & e intertextuales \\
\hline
\end{tabular}

Fuente: Olave-Arias, Rojas-García, Cisneros-Estupiñán, 2013.

tienen un índice mayor de deserción, en las universidades este riesgo disminuye. No obstante, "créditos y apoyos (académicos, financieros y de otra índole) disminuyen el riesgo de deserción, independientemente del carácter de la IES" (p. 49).

Para Salcedo (2010), condiciones institucionales como: escasos recursos y defectos de organización, superpoblación universitaria, deficiencias docentes, falta de apoyo organizado al estudiante (bienestar estudiantil, becas, entre otros), currículos inadecuados y carencia de información estadística se constituyen en factores de riesgo para la deserción. En esta dirección argumenta Rojas (2009) cuando muestra que la deserción es un "indicador de la capacidad de gestión universitaria en un mundo educativo más complejo y de altas exigencias (...) que requieren de una estructura organizacional de gestión ágil, oportuna, eficiente y eficaz" (p. 81). Este autor esboza la crisis de un mundo universitario que no responde a las demandas reales del contexto por modelos pedagógicos y de evaluación obsoletos; planes de estudio que no interlocutan con las expectativas de los estudiantes; estilos docentes y administrativos "rígidos" y "poco amistosos". Estos patrones académicos arcaicos, exigentes en la forma y poco atractivos para la vida, contrastan de modo negativo con la devaluación de las credenciales educativas (Martín-Barbero, 2003, citado en Rojas, 2009) en un contexto en el que el esfuerzo para la educación no se compensa socialmente, pues, la oferta laboral es insuficiente.
De manera consonante, Londoño (2013) encuentra como factores institucionales que inciden en la deserción de la Corporación Lasallista: insatisfacción con estrategias de permanencia y reingreso implementadas por la IES, percepción negativa del nivel académico de los docentes, ausentismo profesoral y ubicación distante de la sede. En ese orden.

Por su parte, el perfil protector arrojado por el estudio de la Universidad Nacional plantea, como uno de los cuatro factores principales para la permanencia, un alto nivel de integración social e institucional vinculado a: ofertas laborales dentro de la universidad, vínculos fuertes con pares y maestros, ofertas deportivas, culturales y de salud. Merece especial atención el hecho de que la integración se mantenga tras la graduación como sentido de pertenencia y vinculación con los docentes en el marco de conformación de comunidad académica.

Rojas (2009) y Pineda-Báez y sus colegas (2011) enfatizan en el rol del docente tanto referido a la calidad humana, académica y pedagógica como a los efectos de ambientes represivos frente a las manifestaciones culturales de los jóvenes.

\section{Retención, permanencia, prevención de la deserción}

La identificación de fenómenos asociados a la deserción abrió el camino al diseño de programas de retención. El estudio del Ministerio de Educación (2009) recomienda a tales programas centrarse en 
aquellas variables que, pueden ser intervenidas de forma directa por la institución, y son identificadas como determinantes fuertes del riesgo de deserción o del aumento en la graduación. Dentro de las medidas propuestas están: fomento de programas de apoyo académico, transferencia entre programas, mejoramiento de mecanismos de regulación en el ingreso de estudiantes que cursan a la par otras carreras, incremento y mejoría de la información sobre los programas, creación de programas de ayuda financiera para estudiantes vulnerables en su condición económica o provenientes de otras ciudades y orientación vocacional previa a la inscripción al programa.

Además de estas medidas, promovidas de manera diferencial en los estudios revisados, se encuentran también otras. En la Universidad Nacional (Durán et al., 2007), además, se propone el establecimiento de un sistema de monitoreo de la deserción, consejerías, programas de transición, involucramiento de padres, nivelación académica, acciones para la integración a las dinámicas universitarias y conformación de redes de apoyo.

Los documentos del MEN (2008 y 2009) retomando estudios como el de Castaño y sus colegas (2006) recomiendan estrategias de bienestar como: mejoramiento de la orientación para la vida universitaria y la inserción académica; orientación para la inserción en el mercado laboral y la continuidad académica una vez terminado el programa; creación del observatorio laboral para la educación. El estudio de la UIS (Cardozo-Ortiz, 2011) resalta estrategias para el seguimiento académico, como la tutoría de pares y un Programa de Asesoría para el Mejoramiento del Rendimiento Académico (Pamra). Los estudios de Pineda-Báez y colegas (2011) y Suárez-Montes y Díaz-Subieta (2015) proponen cursos de nivelación y remediales para reforzar procesos de aprendizaje, y acompañamiento psicológico para el fortalecimiento de capacidades y manejo de problemas de salud mental. El estudio de Montes y sus colegas (2010) responde a la mayor incidencia de la deserción temprana en su institución por lo cual focaliza apoyos académicos y financieros en los primeros semestres y en población vulnerable, promueve la continuidad de la comprensión del fenómeno y realiza acciones de fortalecimiento académico (técnicas de estudio, consultorio matemático y flexibilización curricular).

A partir de los hallazgos en la Corporación Lasallista, Londoño (2013) propone acciones para disminuir el ausentismo profesional y mejorar condiciones adversas de contratación docente. Barragán y Patiño (2013), a partir de su diagnóstico de frustración juvenil y desconocimiento institucional de la juventud, recomiendan profundizar los conocimientos sobre la realidad sociocultural de los estudiantes para complementar los estudios estadísticos y permitir comprensiones más holísticas de la deserción.

El estudio sobre el papel del docente en programas de retención, realizado en 23 instituciones de educación superior en Colombia, refiere una tipificación de los programas orientados a facilitar el tránsito estudiantil, según sus énfasis (Swail, Redd y Perna, 2003 citados en Pineda-Báez, Pedraza-Ortiz y Moreno, 2011): programas de promoción y admisión, servicios académicos, apoyo financiero, programas relacionados con el currículo y la instrucción, y programas de servicio estudiantil. Esta tipificación estructura de manera ordenada medidas posibles centradas en aspectos institucionales.

Los programas de promoción y admisión constan de acciones informativas y de orientación para llegar a la población meta del programa y ajustar los valores de los recién llegados a las apuestas institucionales. Buscan una transición fluida de la educación secundaria al medio universitario por medio de orientación vocacional adecuada e información precisa sobre el programa. Ponen como ejemplo visitas promocionales a instituciones de educación media, semanas de inducción o cursos introductorios.

Los programas de servicios académicos fortalecen las capacidades académicas de los estudiantes en procesos lecto-escriturales y de pensamiento lógicomatemático por medio de tutorías, consejerías, cursos de nivelación y vacacionales, y monitorías. Estas últimas, con doble función pues además fortalecen económicamente a quienes las realizan.

Los programas de apoyo financiero se orientan a facilitar la financiación de los estudios y subvencionar gastos de supervivencia: créditos educativos gubernamentales, institucionales y bancarios; refinanciación de matrículas; préstamos condonables; becas institucionales; auxilios empresariales; subsidios de alimentación, vivienda, libros y materiales; ofertas laborales dentro de la institución y pasantías.

Los servicios estudiantiles o de bienestar promueven la vinculación de los estudiantes con su medio para buscar una mejor integración social. En Colombia están consagrados por la Ley 30 de 1992 (III, 117-118) que los define como el conjunto de actividades orientadas al desarrollo físico, psicoafectivo, espiritual y social de estudiantes, docentes y personal administrativo, a las cuales debe destinarse por lo menos un $2 \%$ del presupuesto institucional.

Otro grupo de acciones, poco explorado en las investigaciones sobre retención, está conformado por aquellas que examinan la calidad de los currículos y 
prácticas pedagógicas buscando "generar ambientes óptimos que incidan en la motivación de los estudiantes para continuar vinculados a los programas" seleccionados (Swail, Redd y Perna, 2003, citados en Pineda-Báez et al., 2011, p. 124). Al respecto es necesario hacer énfasis en que la motivación es catalogada (Berg, 2010, citado en Rodríguez et al., 2013) como el factor clave para el ingreso y la permanencia de estudiantes de bajos recursos en el sistema de educación secundaria.

En relación con el componente pedagógico se identifican cinco factores (Kuh, Kinzie, Schuh y Whitt, 2005, citados en Pineda-Báez et al., 2011) que favorecen la permanencia: el reto académico que desafía al estudiante intelectual y creativamente; el aprendizaje activo y colaborativo desarrollado con pares; la interacción entre estudiantes y docentes en el ámbito investigativo más allá del aula de clase; oportunidades educativas enriquecedoras estimulantes de vínculos con el contexto social, y ambientes que fomenten la buena realización de actividades académicas y el fortalecimiento de las relaciones sociales.

Dentro de las estrategias que el estudio en mención favorece se encuentran: conformación de comunidades de aprendizaje que estimulen el crecimiento intelectual del estudiante; fortalecimiento en los maestros de una actitud de disponibilidad para la atención a los estudiantes y la promoción de la persistencia; un manejo relacional basado en la confianza pues esta incrementa la eficiencia de los procesos formativos, la motivación al aprendizaje, un ambiente propicio para la discusión y el respeto de diferentes puntos de vista, la toma informada de decisiones, la autodeterminación y autovaloración, y la comunicación efectiva. Razones que hacen necesario el desarrollo de competencias comunicativas y sociales por parte de los docentes (saber escuchar, aumentar la motivación, ser asertivo al preguntar, favorecer el desarrollo afectivo).

Este estudio invita a las instituciones a una reflexión que vale la pena citar en extenso:

[...] la solución al problema de abandono escolar a nivel de educación superior requiere de la constante reflexión de las instituciones sobre sus propios procesos, y esto involucra, entre muchos otros aspectos, el quehacer pedagógico, la manera como el docente se relaciona con el educando y lo vincula en sus prácticas de investigación e innovación y las acciones que emprenda la institución para favorecer el enriquecimiento y modernización de sus prácticas, de manera que permitan al docente reinventarse [...]. (Pineda-Báez et al., 2011, p. 126).
Para cerrar, el citado estudio advierte sobre la necesidad de que los planes de retención sean elaborados y ejecutados con amplia participación docente para enriquecer el debate y dar pie a "transformaciones consensuadas de los programas (...) fortaleciendo los mecanismos de comunicación y socialización de la institución" (Pineda-Báez et al., 2011, p. 132) para que los docentes informados se interesen en participar en las acciones propuestas.

\section{Conclusiones}

La revisión documental permitió establecer dos tendencias en los estudios del periodo analizado. Se evidencia un desplazamiento desde una mirada cuantitativa hacia aproximaciones cualitativas del fenómeno, que coincide con el traslado de la atención desde la deserción hacia la identificación de acciones para su prevención sostenible.

En parte importante de los estudios resalta la preocupación por el carácter estructural de la deserción en Colombia, con mayor incidencia en estratos socioeconómicos bajos, en los que los factores económicos se conjugan y reflejan en capital cultural y simbólico precario para la formación académica. Quienes vienen de mejores condiciones económicas tienen posibilidad de un tránsito más fluido por las habilidades requeridas en la universidad (formas de argumentación, medios de acceso a información, capacidad lectoescritural, etc.).

El reforzamiento de estas habilidades como responsabilidad institucional es uno de los avances que evidencian las estrategias de retención y se convierte en paso importante para deconstruir la relación establecida en la cultura, entre éxito académico y dones o aptitudes. Al respecto, algunos estudios enfatizan la dimensión subjetiva del éxito académico que, en estudiantes de bajos recursos, remite a la motivación como elemento fundamental para superar las adversidades de contextos e historias de exclusión. Tal motivación está estrechamente relacionada con el rol docente y sus habilidades comunicativas. Ello invita a pensar sobre el atractivo del estudio en los proyectos de vida de los jóvenes y a reflexionar sobre las formas de relacionamiento y autocomprensión que impulsan instituciones universitarias y docentes, en aras de un diálogo penetrante con el mundo de la vida y con las percepciones de los jóvenes acerca de su contexto y del tiempo que habitan.

A pesar del carácter estructural excluyente de la educación superior en Colombia, el fenómeno de la deserción dista de ser homogéneo. Se trata de un fenómeno contextual que debe estudiarse con 
precisión para dar respuestas adecuadas. En este sentido resalta la consideración de la deserción como indicador de la gestión institucional, pues es un fenómeno vinculado de forma estrecha con el cumplimiento de la misión de las instituciones de educación superior.

Los estudios sobre deserción deben preocuparse por una conceptualización rigurosa que permita identificar con amplia certeza los determinantes locales del fenómeno y establecer acciones adecuadas para su disminución. Para ello es fundamental la adecuada gestión de la información sobre los estudiantes, con el fin de establecer causales y correctivos consistentes.

\section{Referencias}

Ariza, S. y Marín, D. (2009). Factores intervinientes en la deserción escolar de la Facultad de Psicología, Fundación Universitaria Los Libertadores. Tesis Psicológica, 4, 72-85. Recuperado de http://www. redalyc.org/pdf/1390/139013586006.pdf

Asociación Colombiana de Universidades -ASCUN. (2007). Políticas y estrategias para la Educación Superior de Colombia 2006-2010. Bogotá: Coarcas.

Barragán, D. y Patiño, L. (2013). Elementos para la comprensión del fenómeno de la deserción universitaria en Colombia. Más allá de las mediciones. Cuadernos latinoamericanos de Administración, 11 (16), 55-66. Recuperado de http://www.redalyc.org/articulo. oa?id=409633954005

Bean, J. (1980). Student attrition, intentions and confidence. Research in Higher Education, 17, 291-320.

Berg, G. (2010). Low-income students and the perpetuation of inequality: Higher Education in America. ASHGTE. Recuperado de http://books.google.com.co/books?i $\mathrm{d}=76 \mathrm{PCneys} 9 \mathrm{UEC} \& \mathrm{pg}=\mathrm{PA} 41 \& \mathrm{dq}=$ low-income + univ ersity+students\&hl=es\&sa=X\&ei=06J5UcTgIY7c8w SWroCQDw\&ved=0CDUQ6AEwAQ\#v=onepage \&q= low-income $\% 20$ university $\% 20$ students\&f=false

Bourdieu, P. (2000). Capital cultural, escuela y espacio social. México: Siglo XXI.

Bravo, M. y Mejía, A. (2010). Los retos de la educación superior en Colombia: una reflexión sobre el fenómeno de la deserción universitaria. Revista de Educación en Ingeniería, 5 (10), 85-98. Recuperado de www.acofi. edu.co

Caicedo, J. y Castillo, E. (2008). Indígenas y afrodescendientes en la universidad colombiana: nuevos sujetos, viejas estructuras. Cuadernos Interculturales, 6 (10), 62-90. Recuperado de http://www.redalyc.org/articulo.oa?id=55261005
Cardozo-Ortiz, C. (2011). Tutoría entre pares como una estrategia pedagógica universitaria. Educación y Educadores, 14 (2), 309-325. Recuperado de http:// www.redalyc.org/articulo.oa?id=83421404006

Castaño, E., Gallón, S., Gómez, K. y Vásquez, J. (2006). Análisis de los factores asociados a la deserción y graduación estudiantil universitaria. Lecturas de Economía, 65, 9-36. Recuperado de http://www.alfaguia.org/ alfaguia/files/1319760796_15.pdf

Castro, B. y Rivas, G. (2006). Estudio sobre el fenómeno de la deserción y retención escolar en localidades de alto riesgo. Sociedad Hoy, 011, 35-72.

Centro de Estudios Económicos - CEDE-, Universidad de Los Andes. (2007). Investigación sobre deserción en las instituciones de educación superior en Colombia [Informe final]. Bogotá: Universidad de los Andes. Recuperado de http://studylib.es/doc/7619308/ informe-t\%C3\%A9cnico-del-cede---ministerio-deeducaci $\% \mathrm{C} 3 \% \mathrm{~B} 3 \mathrm{n}$

Centro de Estudios Económicos - CEDE-, Universidad de Los Andes. (2014). Determinantes de la deserción. [Informe mensual sobre el soporte técnico y avance del contrato para garantizar la alimentación, consolidación, validación y uso de la información del SPADIEs]. Bogotá: Universidad de los Andes. Recuperado de https://www. mineducacion.gov.co/sistemasdeinformacion/1735/ articles-254702_Informe_determinantes_desercion.pdf

Donoso, S. y Schiefelbein, E. (2007). Análisis de los modelos explicativos de retención de estudiantes en la universidad: una visión desde la desigualdad social. Estudios Pedagógicos, 33 (1), 7-27.

Durán, D., Pérez-Almonacid, R., Rodríguez, A., Reverón, C. y Pinto, M. (2007). Cuestión de Supervivencia. Graduación, deserción y rezago en la Universidad Nacional de Colombia. Bogotá: Universidad Nacional de Colombia. Recuperado de http://www.bienestar.unal.edu.co/wpcontent/uploads/2015/05/cuestion_supervivencia.pdf

Ethington, C. (1990). A psychological model of student persistence. Research in Higher Education, 31 (3), 266-269.

Fishbein, M. y Ajzen, I. (1975). Belief, attitude, intention, and behavior: An introduction to theory and research. Reading, MA: Addison-Wesley.

Giovagnoli, P. (2002). Determinantes de la deserción y la graduación universitaria: una aplicación utilizando modelos de duración. Documentos de trabajo 37. Universidad Nacional de la Plata.

Kuh, G., Kinzie, J., Schuh, J. y Whitt, E. (2005). Student success in college. Creating conditions that matter. San Francisco, CA: Jossey-Bass, A Wiley Imprint. 
Londoño, L. (2013). Factores de riesgo presentes en la deserción estudiantil en la Corporación Universitaria Lasallista. Revista Virtual Universidad Católica del Norte, 38. 183-194. Recuperado de http://revistavirtual.ucn. edu.co/index.php/RevistaUCN/article/view/413

Lopera, C. (2008). Determinantes de la deserción universitaria en la Facultad de Economía. Serie Documentos. Borradores de Investigación, 95. Bogotá: Universidad del Rosario. Recuperado de http://www.alfaguia.org/ alfaguia/files/1318975055Determinantes\%20de\%20 la $\% 20$ desercion $\% 20$ universitaria $\% 20$ en $\% 20$ la $\% 20$ facultad $\% 20 \mathrm{de} \% 20$ economia $\% 20$ universidad $\% 20$ del\%20rosario.pdf

Malagón, L., Soto L. y Eslava, P. (2007). La deserción en la Universidad de los Llanos (1998-2004). Revista Orinoquía, 11, 22-40. Recuperado de orinoquia. unillanos.edu.co/index.php/orinoquia/article/ view/166/604

Martín-Barbero, J. (2003). Oficio de cartógrafo. Travesías latinoamericanas de la comunicación en la cultura. México: FCE

Mateus, M., Herrera, C., Perilla, C., Parra, G. y Andersen, V. (2011). Factores presentes en la deserción en la Facultad de Psicología, Universidad de San Buenaventura, sede Bogotá 1998-2009. Psychologia. Avances de la disciplina, 5 (1), 121-133. Recuperado de http://www.redalyc. org/pdf/2972/297224114011.pdf

Medellín, W. (2010). Contrastación de dos modelos motivacionales de autodeterminación para predecir la deserción en universitarios. Acta colombiana de psicología, 13 (2), 57-68. Recuperado de http://editorial.ucatolica. edu.co/ojsucatolica/revistas_ucatolica/index.php/ acta-colombiana-psicologia/article/view/370/375

Ministerio de Educación Nacional — MEN. (2008). Deserción estudiantil en la educación superior colombiana. Elementos para su diagnóstico y tratamiento. Bogotá: Imprenta Nacional. Recuperado de http://www.mineducacion.gov.co/sistemasdeinformacion/1735/articles-254702_diagnostico_desercion.pdf

Ministerio de Educación Nacional — MEN. (2009). Deserción estudiantil en la educación superior colombiana. Metodología de seguimiento, diagnóstico y elementos para su prevención. Bogotá: Imprenta Nacional. Recuperado de http://www.mineducacion.gov.co/ sistemasdeinformacion/1735/articles-254702_libro_ desercion.pdf

Montes, I., Almonacid, P., Gómez, S., Zuluaga, F. y Tamayo, E. (2010). Análisis de la deserción estudiantil en los programas de pregrado de la universidad EAFIT.
Cuadernos de Investigación, 81. Documento 81-042010. Recuperado de publicaciones.eafit.edu.co/index.php/ cuadernos.../article/.../1145

Olave-Arias, G., Rojas-García. I. y Cisneros-Estupiñán, M. (2013). Deserción universitaria y alfabetización académica. Revista Educación y educadores, 16 (3), 455-471. Recuperado de http://www.redalyc.org/ pdf/834/83429830004.pdf

Osorio, A., Bolancé, C. y Castillo-Caicedo, M. (2012). Deserción y graduación estudiantil universitaria: una aplicación de los modelos de supervivencia. Revista Iberoamericana de Educación Superior, 3 (6), 31-57. Recuperado de https://ries.universia.net/article/ viewFile/72/130

Patiño, L. y Cardona, A. (2015). Revisión de algunos estudios sobre la deserción estudiantil universitaria en Colombia y latinoamérica. Theoria, 21 (1), 9-20. Recuperado de www.ubiobio.cl/miweb/webfile/media/194/v/ v21-1/1.pdf

Pineda-Báez, C., Pedraza-Ortiz, A. y Moreno, I. (2011). Efectividad de las estrategias de retención universitaria: la función del docente. Educación y Educadores, 14 (1), 119-135. Recuperado de http://www.redalyc.org/ articulo.oa?id=83418921008

República de Colombia (1991). Constitución Política de Colombia. Recuperada de http://www.constitucioncolombia.com/

Rodríguez, L. (2011). Estudio sobre deserción estudiantil en los programas de Educación de la Católica del Norte Fundación Universitaria. Revista Virtual Universidad Católica del Norte, 33, 328-355. Recuperado de http:// revistavirtual.ucn.edu.co/index.php/RevistaUCN/ article/view/21

Rodríguez, M., Posada, M., Estrada, P.y Velásquez, M. (2013). Población con riesgo de abandono universitario. Una aproximación desde la prevención. Ponencia presentada en Tercera Conferencia latinoamericana sobre el abandono de la educación superior. Recuperada de http:// www.alfaguia.org/www-alfa/images/ponencias/clabesIII/LT_1/ponencia_completa_71.pdf

Rojas, H. (2009). El abandono de los estudios: deserción y decepción de la juventud. Hologramática, 4 (10), 75-94. Recuperado de https://dialnet.unirioja.es/servlet/ articulo?codigo $=3735200$

Rojas, M. y González, D. (2008). Deserción estudiantil en la Universidad de Ibagué, Colombia: una lectura histórica en perspectiva cuantitativa. Revista del Instituto de Estudios en Educación, 9, 70-83. Recuperado de http:// www.redalyc.org/articulo.oa?id=85312286006 
Sánchez, F. y Márquez, J. (2012). La Deserción en la Educación Superior en Colombia durante la primera década del siglo xxi: ¿Por qué ha aumentado tanto? Cuadernos Cede, 31. Recuperado de http://www. colombiaaprende.edu.co/html/micrositios/1752/ articles-324998_recurso_1.pdf

Sánchez, A. y Otero, A. (2012). Educación y reproducción de la desigualdad en Colombia. Reportes del Emisor, Investigación e Información Económica. No. 154. Banco de la República.

Spady, W. (1970). Dropouts from higher education: an interdisciplinary review and synthesis. Interchange, 1 (1), 64-85.

Stratton, L., O’Toole, D. y Wetzel, J. (2008). A multinomial logit model of college stopout and dropout behaviour. Economics of Education Review, 27, 319-331.

Suárez-Montes, N. y Díaz-Subieta, L. (2015). Estrés académico, deserción y estrategias de retención de estudiantes en la educación superior. Revista de Salud Pública, 17 (2), 300-313. Recuperado de http://dx.doi. org/10.15446/rsap.v17n2.52891
Swail, W., Redd, K. y Perna, L. (2003). Retaining minority students in higher education: A framework for success. ASHE-ERIC Higher Education Report, 30 (2). Jossey-Bass Higher and Adult Education Series (ERIC Document Reproduction Service N ED483024).

Tinto, V. (1975). Dropout from Higher Education: A theoretical synthesis of recent research. Review of Educational Research, 45, 89-125.

Tinto, V. (1982). Limits of theory and practice in student atrittion. Journal of Higher Education, 53 (6), 687-700.

Tinto, V. (1987). Leaving college: Rethinking the causes and cures of student attrition. Chicago: University of Chicago Press.

Tinto, V. (1989). Definir la deserción: una cuestión de perspectiva. Revista Educación Superior, 18 (71).

Torres, L. (2012). Retención estudiantil en la educación superior. Revisión de la literatura y elementos de un modelo para el contexto colombiano. Bogotá: Universidad Javeriana. Recuperado de http://www.javeriana.edu. co/documents/15838/273636/Retenci\%C3\%B3n Estudiantil2012.pdf/124fdba5-2318-432a-8e9f126a2501c229 